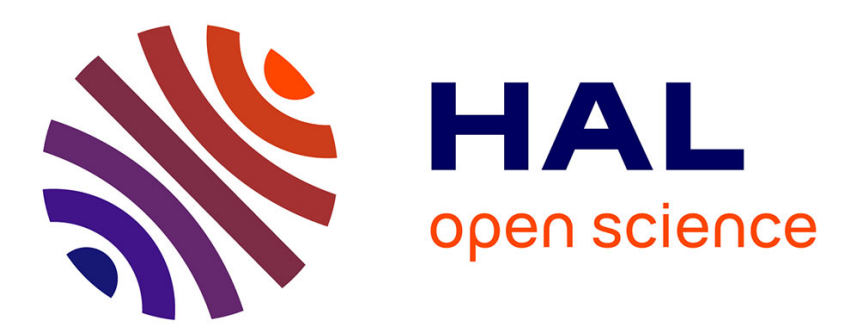

\title{
La traduction de la demande d'aide sociale: le cas du handicap et de l'insertion des jeunes
}

Marie-Christine Bureau, Lea Lima, Barbara Rist, Christophe Trombert

\section{To cite this version:}

Marie-Christine Bureau, Lea Lima, Barbara Rist, Christophe Trombert. La traduction de la demande d'aide sociale: le cas du handicap et de l'insertion des jeunes. Revue française d'administration publique, 2013, $\mathrm{n}^{\circ} 145$, pp. 175-188. 10.3917/rfap.145.0175 . halshs-00881022

\section{HAL Id: halshs-00881022 \\ https://shs.hal.science/halshs-00881022}

Submitted on 7 Nov 2013

HAL is a multi-disciplinary open access archive for the deposit and dissemination of scientific research documents, whether they are published or not. The documents may come from teaching and research institutions in France or abroad, or from public or private research centers.
L'archive ouverte pluridisciplinaire HAL, est destinée au dépôt et à la diffusion de documents scientifiques de niveau recherche, publiés ou non, émanant des établissements d'enseignement et de recherche français ou étrangers, des laboratoires publics ou privés. 


\section{La traduction de la demande d'aide sociale : le cas du handicap et de l'insertion des jeunes}

Marie-Christine Bureau, Chargée de recherche au CNRS, Laboratoire Interdisciplinaire de Sociologie Economique (LISE), courriel : marie-christine.bureau@cnam.fr

Léa Lima, Maître de conférences au Conservatoire National des Arts et Métiers (CNAM), LISE

Barbara Rist, Maître de conférences au CNAM, LISE

Christophe Trombert, Maître de conférences à l'Université de Lyon 2, Centre Max Weber

\section{Résumé}

En accumulant des données sur deux dispositifs d'attribution des droits sociaux (aides aux jeunes et droits liés au handicap), nous examinons quel est le statut réservé au demandeur, puis les logiques bureaucratiques et morales qui président au tri des demandes par les acteurs des deux dispositifs. Nous nous interrogeons ensuite sur les marges de manœuvre effectives des usagers dans la co-construction de la demande ainsi que sur les façons dont s'élabore la connaissance des besoins auxquels il s'agit de répondre. Cette comparaison montre qu'il n'y a pas une, mais des «magistratures sociales", plus ou moins participatives, plus ou moins conditionnelles et discrétionnaires, selon le type de bénéficiaires.

Mots-clé : droits sociaux -usagers - magistrature sociale - jeunes -handicap 


\section{Introduction}

Ce que l'on appelle communément individualisation des droits sociaux regroupe en fait une série de processus cohérents entre eux. La catégorisation administrative des publics, formée $a$ priori, aurait, selon Pierre Rosanvallon (1995), trouvé ses limites au fil des dispositifs. Les catégories de l'action publique, comme l'insertion, ont pris un tour de plus en plus interprétable en se tournant vers l'avenir d'un projet personnalisé, supposant une logique procédurale de mise en adéquation des aides et des bénéficiaires. L’individualisation ne s'arrête pas à la prise en compte du jugement de proximité sur les personnes et leur état : le jugement s'étend aux situations, si bien que les intermédiaires chargés d'une «magistrature sociale » (Astier, 1997) se doivent non pas tant de vérifier la conformité des cas aux règles que de s'enquérir de la pertinence de l'aide au regard d'une biographie et d'une trajectoire. Outre la recherche d'un mode plus équitable de répartition des ressources, la personnalisation de la protection sociale vise une plus grande implication des bénéficiaires, à la fois subjective et pratique, dans la définition de leurs besoins et des solutions à leurs problèmes.

Jusqu'où prendre en compte l'expression singulière de chacun tout en visant un objectif d'équité, dans un contexte de massification des demandes d'aides sociales? L'individualisation des droits va-t-elle concrètement de pair avec une plus grande reconnaissance de la capacité des usagers à définir leurs propres besoins ? Ou bien relève-telle d'une «nouvelle figure de la domination », à travers la double injonction d'authenticité (dire la vérité sur soi) mais aussi d'énonciation d'un projet, adressée aux demandeurs (Martucelli, 2004)?

L'observation de deux «magistratures sociales» répondant au référentiel de l'individualisation, les CDAPH (Commission des droits et de l'autonomie des personnes handicapées) qui attribuent, entre autres, les prestations de compensation du handicap ( $\mathrm{PCH})$ et les FAJ (Fonds d'aide aux jeunes), dans 4 départements différents pendant une année entière, montre que ces questions sont au cœur des préoccupations des acteurs de terrain. Elle nous permet de souligner les contraintes qui pèsent sur l'organisation de l'expression des usagers dans le traitement des demandes et les limites qui en découlent en termes de coconstruction de la demande. Nous nous situons ainsi à un niveau meso, entre une analyse de l'action publique qui porterait sur la transformation des référentiels ou des injonctions (à la responsabilisation, à l'autonomie, à la participation) et une sociologie de l'interaction au guichet (Dubois, 2010a ; Siblot, 2006 ; Spire, 2008) pour saisir la manière dont l'agencement et les conditions concrètes de fonctionnement des magistratures sociales structure les pratiques des street-level bureaucrats (Lipsky, 1980) et leur relation aux usagers. 
Encart méthodologique

Cette contribution s'appuie sur une enquête qui s'est étalée entre juin 2009 et septembre 2010 dans deux Maisons départementales des personnes handicapées (MDPH), à Paris et à Saint-Etienne, et deux Fonds départementaux d'aide aux jeunes (FAJ) en région parisienne.

Créée par la loi de 2005, la PCH vise à compenser les conséquences du handicap quelles que soient l'origine et la nature de la déficience, l'âge ou le mode de vie de la personne, afin de lui assurer une autonomie sociale et professionnelle. Elle est attribuée, après évaluation, par une équipe pluridisciplinaire des besoins de la personne (aides humaines, techniques, animalière, etc.), en tenant compte de son projet de vie. Les MDPH assurent l'organisation de la commission et le suivi de la mise en œuvre de ses décisions, ainsi que la gestion du fonds départemental de compensation du handicap. D’une façon générale, la CDAPH prend l'ensemble des décisions relatives aux droits des personnes handicapées, notamment en matière d'attribution de prestations et d'orientation. Elle est compétente pour l'ensemble des décisions individuelles d'allocation ou de prestation, de reconnaissance de la qualité de travailleur handicapé, d'orientation scolaire, médico-sociale, professionnelle et d'attribution de cartes d'invalidité et de stationnement.

Le FAJ est instauré par la loi favorisant le retour à l'emploi et la lutte contre l'exclusion professionnelle du 19 décembre 1989. Facultatif dans un premier temps, il est devenu obligatoire en 1992 puis passé sous la compétence exclusive des conseils généraux en 2004.

Le FAJ apporte une aide financière "ponctuelle» aux jeunes de 18 à 25 ans "sans ressources ou avec des ressources très faibles ». Les objets d'aide les plus couramment répertoriés sont l'aide à la subsistance (repas, nuitées), l'aide à la mobilité, l'aide à la formation, l'aide au logement, et l'aide à la recherche d'emploi. Est prévue dans les textes une procédure d'urgence qui permet de soumettre une demande directement au secrétariat du FAJ qui peut la traiter dans les 48 heures (au lieu de 15 jours, trois semaines pour une demande par la voie normale). 135000 aides financières ont été distribuées en 2008 sur l'ensemble des départements pour un montant de 193 euros en moyenne. Dans ce cas les magistratures sociales prennent la forme de commissions d'attribution du FAJ qui sont chargées d'examiner les dossiers de demande d'aide financière de jeunes en difficultés de moins de 25 ans. Ces commissions dont le budget est voté chaque année par les Conseils Généraux se fixent leurs propres règles mais l'économie générale du dispositif repose sur le dossier de demande d'aide complété par le travailleur social (dans 70\% un conseiller 
d'insertion en mission locale) et qui émet un avis sur la pertinence de la demande. Elles mettent en présence les financeurs avec une équipe technique dont la composition est variable mais au cœur de laquelle se trouvent les professionnels des missions locales pour l'insertion des jeunes.

Sur chacun des terrains, nous avons procédé à une série d'observations ethnographiques des délibérations des commissions ainsi qu'à des entretiens avec les principaux protagonistes.

Nous examinerons dans un premier temps le statut qui est réservé au demandeur ou à l'usager dans les deux secteurs du point de vue de leurs dispositions à la fois législatives et règlementaires. Puis nous verrons les logiques bureaucratiques et morales qui président au tri des demandes par les acteurs à la fois professionnels et institutionnels des deux dispositifs. Nous prolongerons ensuite cette incursion dans les pratiques afin d'évaluer les marges de manœuvre des usagers dans la co-construction de la demande.

\section{Le statut de la demande : un droit conditionnel ?}

La comparaison entre l'attribution des FAJ et celle des droits sociaux liés au handicap fait apparaître des situations contrastées quant au statut de la demande : à l'inverse de la compensation du handicap, affirmée comme un droit, le FAJ apparaît d'abord comme un outil au service des professionnels.

\subsection{Une aide prescrite $v s$ un droit à compensation}

Le fonds d'aide aux jeunes s'insère dans des politiques sociales de jeunesse qui n'accordent globalement qu'une place marginale aux droits sociaux subjectifs pour leur préférer un système plus complexe de sécurisation des trajectoires par des mesures dont les professionnels de l'insertion des jeunes contrôlent peu ou prou la prescription (Lima, 2008). De ce fait, même si les professionnels de l'accompagnement social et professionnel ne peuvent pas, en théorie, refuser de monter un dossier si un jeune en formule le souhait, le FAJ reste considéré avant tout comme un outil au service de l'accompagnement des jeunes. L'inscription du jeune dans une démarche institutionnalisée d'accompagnement est une condition d'accès à ce fonds ${ }^{\mathrm{i}}$ dont ni l'Etat, ni les Conseils Généraux ne font la publicité auprès du public. Il est donc plus juste de dire que le référent du jeune se trouve à l'origine de la demande, jugeant de l'opportunité de monter un dossier à un moment donné de son travail d'accompagnement. En ce sens, l'aide financière du FAJ se propose, voire se prescrit, plus qu'elle ne se demande. 
Dans le champ du handicap en revanche, la loi du 11 février 2005 stipule que « la personne handicapée a droit à la compensation des conséquences de son handicap » et que «cette compensation consiste à répondre à ses besoins »: c'est même là l'une des innovations majeures de la loi. En outre, la gamme de besoins considérés apparaît a priori très étendue $\mathrm{e}^{\mathrm{ii}}$. Il ne s'agit pas pour autant d'une attribution automatique. Selon la loi, le plan de compensation doit être élaboré en considération des aspirations de la personne handicapée, exprimées par exemple dans son projet de vie. Au sein du formulaire de demande, organisé par type de droit ou de prestation (allocation, reconnaissance de la qualité de travailleur handicapé, carte d'invalidité, prestation de compensation du handicap etc.), le projet de vie constitue la seule rubrique du dossier permettant une réponse ouverte, avec l'invite suivante : « Précisez, si vous le souhaitez, vos attentes et besoins en termes de communication, santé, scolarité, formation, travail, logement, vie quotidienne, vie affective, vie familiale, loisirs ... ». Il n'est pas obligatoire de le remplir et cela ne constitue en rien une condition pour l'accès aux droits. D'après le rapport d'activité des MDPH en 2008, la formulation des projets de vie varie d'ailleurs beaucoup d'une MDPH à l'autre mais reste rare : dans la moitié des départements, le projet de vie n'est renseigné que dans $10 \%$ des demandes ${ }^{\mathrm{iii}}$.

\subsection{Quels moyens légaux d'intervention de l'usager?}

Les textes précisent en outre : « la personne adulte handicapée, le cas échéant son représentant légal, les parents ou le représentant légal de l'enfant ou de l'adolescent handicapé sont consultés par la commission des droits et de l'autonomie des personnes handicapées. Ils peuvent être assistés par une personne de leur choix ou se faire représenter » (article L241-7 du code de l'action sociale et des familles). En cas de refus d'une prestation par la commission ou d'un plan de compensation jugé insuffisant ou inadapté par le demandeur, celui-ci dispose de possibilités de recours gracieux ou contentieux. En 2008, les recours représentent en moyenne $2 \%$ des décisions prises, dont les $2 / 3$ sont des recours gracieux. Pour limiter les contentieux, des services de conciliation et de médiation ont été mis en place : en 2008, 84\% des MDPH disposent d'un conciliateur et $62 \%$ ont nommé un médiateur. Dans l'une des MDPH observées, la commission plénière auditionne les personnes concernées avant de délibérer sur l'attribution des droits, en particulier dans les cas de recours gracieux. Dans l'autre, les usagers sont plus souvent reçus au sein de commissions restreintes, soit à leur demande, soit lorsqu'ils ont fait un recours ou encore lorsque les équipes pluridisciplinaires ressentent le besoin d'un complément d'information pour instruire le dossier. Les personnes handicapées bénéficient en outre d'un mode d'intervention indirecte 
par la voix des associations qui les représentent et composent un tiers des membres de droit des CDA.

Concernant les FAJ en revanche, les règlements intérieurs départementaux ne prévoient pas que les demandeurs puissent venir défendre eux-mêmes leur demande en premier examen. Sur la vingtaine de règlements intérieurs que nous avons consultés, un seul ouvre cette possibilité comme procédure de recours amiable. Beaucoup proposent une procédure de recours minimale consistant à représenter le dossier devant la même commission. Dans les départements d'enquête, la décision est notifiée au jeune mais une copie du courrier est envoyée à son conseiller en mission locale. Les recours gracieux ou contentieux sont rares.

L'aide financière est rarement versée directement au demandeur, sauf lorsqu'il s'agit d'aide minimale et en urgence pour la subsistance (aide versée alors sous forme de tickets service) ; l'aide est le plus souvent versée à un tiers, par exemple à un organisme de formation, un bailleur ou à un hôtel conventionné par le conseil général. C'est dire le niveau de maîtrise de l'usage dont disposent les individus. Le déficit d'information des jeunes demandeurs sur les règles du dispositif contribue en outre fortement à leur faible implication.

\subsection{Le formatage implicite de la demande dans le champ du handicap}

Si la demande bénéficie d'un statut plus assuré dans le domaine du handicap que dans le cas de l'aide aux jeunes, les modalités d'évaluation des besoins dans ce domaine n'en restent pas moins incertaines, d'autant que le flux des demandes n'autorise guère un traitement approfondi des situations singulières. Dès le débat parlementaire sur la loi de 2005, une question cruciale a été posée à plusieurs reprises : qui va procéder à l'évaluation des besoins et selon quels critères ? Quelques années après la promulgation de la loi, une recherche menée au sein d'une MDPH (Vidal-Naquet, 2009) montre que le mode de traitement des projets de vie reste très peu encadré. L'auteur constate aussi que, même lorsqu'ils sont remplis, la plupart des projets sont fonctionnels, formatés par les prestations existantes. Cela est d'autant plus vrai lorsque la rédaction de ceux-ci a bénéficié du soutien des professionnels qui connaissent bien l'éventail de l'offre et s'efforcent de calibrer les demandes afin d'augmenter leurs chances de prise en considération. Le formatage implicite intervient ainsi dès la première étape d'expression des besoins.

Ainsi, même si la loi prévoit que des préconisations puissent être faites au-delà des rubriques formalisées, cette possibilité reste peu exploitée. Une référente sociale, chargée du deuxième accueil dans une MDPH raconte ainsi : «Je vois souvent les gens tout au début quand ils ne 
savent pas du tout ce qu'ils pourraient demander, à quoi ils peuvent prétendre : "voilà j'ai un problème, quel type de demande je pourrais faire?"Alors moi, je reste, malheureusement, sur les termes de la demande puisque l'on a beaucoup de mal à travailler les préconisations, en particulier sur le secteur adulte. Donc, du coup, j'ai un peu tendance à leur dire "cochez telle demande" alors que je voudrais pouvoir les rassurer et leur dire « expliquez bien votre projet de vie, ce que vous rencontrez comme difficultés, et après, même si vous n'avez pas coché les bonnes cases, il y a des préconisations qui vous seront faites dans le cadre de la compensation ». Dans l'exercice de son métier, la référente souffre beaucoup de ce décalage entre le souci, tel qu'il est exprimé dans la loi, de formuler des préconisations singulières en réponse aux besoins des personnes et la réalité d'un traitement de masse, en grande partie standardisé.

\section{Le tri des demandes}

Le tri des demandes est conditionné dans le FAJ comme dans les MDPH par une double logique: une logique de rationalisation de l'activité dans le contexte de traitement individualisé de masse et une logique morale qui préside à l'établissement d'un certain nombre de critères de justice pour décider d'attribuer ou pas l'aide demandée. Dans un cas, le tri est fortement assuré dès l'instruction, dans l'autre il opère principalement en commission décisionnaire.

\section{$\underline{\text { 2.1. Les effets du traitement individualisé de masse }}$}

Dans les FAJ, les effets de la rationalisation se font sentir dès le moment de l'instruction du dossier par les conseillers. Depuis le premier accueil du jeune jusqu'à l'envoi au service compétent du conseil général, l'instruction est considérée comme un processus chronophage. En effet, hormis les cas d'aides à la subsistance demandées en urgence, le montage du dossier nécessite au moins deux rendez-vous avec le jeune, la nécessité de réunir un nombre important de pièces justificatives, parfois de passer des coups de téléphone à des partenaires. Le dossier demande aussi un effort de rédaction de la part du conseiller, pris sur un temps de travail hors entretien de plus en plus restreint.

Cette conscience aigüe du coût du montage du dossier les porte à évaluer les « chances de réussite » d'une demande avant de se lancer dans la procédure. C'est dans cet état d'esprit qu'ils considèrent donc les demandes des jeunes. Instruire une demande dans laquelle on ne croit pas est une perte de temps. Le montage de la demande passe par une validation du projet d'insertion qui fait l'objet d'un processus assez long : les désirs d'orientation professionnelle 
sont mis à l'épreuve de stages en milieu de travail, de tests psychotechniques, de visites d'entreprises et autres outils dont disposent les professionnels de l'insertion pour confirmer ou infirmer ce que les jeunes présentent comme des choix. Seul le projet «validé » a des chances d'être soutenu par les conseillers qui anticipent en cela pleinement les critères d'appréciation des commissions décisionnaires.

Dans les MDPH, les demandeurs peuvent être reçus par les services d'accueil pour recevoir une aide au montage du dossier mais le tri ne s'effectue pas à ce niveau. Les professionnels de l'accueil sont plutôt en position d'informateurs sur les droits, tandis que l'évaluation des demandes se fait au moment de l'instruction des dossiers par les équipes pluridisciplinaires puis en commissions. La tension entre une approche physiologique du handicap qui se prête à un traitement automatique des demandes et une approche globale et individualisée telle qu'elle est préconisée par la loi, traverse l'ensemble de nos observations: d'un côté, l'application du guide barème conduit à une mesure standardisée du taux d'incapacité ${ }^{\text {iv }}$, même si la loi recommande de ne pas s'en tenir au diagnostic médical ; de l'autre le GEVA ${ }^{\mathrm{v}}$ et le projet de vie synthétisent de manière qualitative l'environnement, la situation de la personne et ses besoins. Cette tension est renforcée par la contrainte de l'afflux des demandes : dans les faits, les MDPH sont débordées par le nombre de dossiers à traiter et les CDAPH n'examinent et ne discutent qu'une infime partie des situations ${ }^{\mathrm{vi}}$.

\subsection{Le tri entre demandes légitimes et illégitimes}

Dans les deux champs étudiés, l'économie morale de ces magistratures sociales fait intervenir plusieurs principes qui conduisent finalement à écarter un nombre important de demandes. Le travail de discrimination des demandes porte à la fois sur un diagnostic et sur un pronostic : le diagnostic dans le registre du besoin qui équivaut à évaluer le degré de fragilité « réelle »de l'individu, voire à débusquer les demandes abusives et, dans une logique d'investissement, le pronostic sur les chances que l'aide octroyée renforce ses capacités à s'intégrer (Lima, 2010a).

Les conseillers d'insertion en mission locale distinguent dans le traitement des demandes, celles qui proviennent d'une démarche spontanée des jeunes et celles qu'ils ont eux-mêmes initiés, de même qu'ils distinguent les demandes formulées par des jeunes lors du premier rendez-vous à la mission locale de celles qui concernent les jeunes en suivi dans cette même structure. Dans les deux cas, les secondes sont toujours jugées plus légitimes et/ou plus réalistes que les premières ${ }^{\mathrm{vii}}$. La demande de FAJ formulée lors d'un premier rendez-vous par le jeune lui-même suscite en effet le soupçon et le doute. Le soupçon, dans le registre de la 
faute, porte sur la qualité de «jeune en difficultés ». Pour les aides «FAJ urgence » (loyer, tickets service), il peut porter sur l'instrumentalisation du dispositif à des fins jugées illégitimes et, au-delà, sur l'instrumentalisation des professionnels eux-mêmes. Ils pointent alors du doigt les jeunes qui démarchent plusieurs conseillers (faisant jouer la concurrence en quelque sorte) pour bénéficier de toutes sortes d'aides, ou encore des jeunes qui demandent alors que les professionnels considèrent qu'ils pourraient exploiter des solutions alternatives pour leur problème. Le glissement vers la demande abusive, celle qui émane d'un « faux » jeune en difficulté n'est pas rare.

Partant de cette méfiance vis-à-vis des demandes spontanées, les conseillers utilisent plusieurs techniques qui visent à mettre à l'épreuve les demandes, voire à les empêcher. Les réticences à l'instruction, pour ne pas dire les refus, ne sont pas rares, d'autant plus que les jeunes ne savent pas qu'ils ont légalement le droit d'exiger que leur demande soit instruite :

«Moi, quand le projet n'est pas cohérent, je ne monte pas le dossier et je l'explique au jeune, « ton projet c'est du n'importe quoi », moi je vais pas perdre de temps à monter un dossier alors que le projet c'est du n'importe quoi ou des frais de formation d'un organisme bidon trouvé sur internet, moi je me refuse à monter ces dossiers [...] donc moi il est hors de question hein que je présente un dossier bâclé » (conseillère en mission locale, FAJ B).

Une autre technique consiste à différer la demande, espérant par la multiplication des rendezvous, faire un tri entre les demandes qui correspondent à un «véritable » besoin et celles qui seraient purement passagères ou opportunistes.

Le doute interroge les chances d'une issue positive à ces parcours soutenus par l'aide publique. La communauté professionnelle de l'insertion des jeunes oppose en effet au modèle de l'assistance celui de l'investissement, dont on doit s'assurer de la «profitabilité ». Dans les cas de demande d'aide pour les frais de formation, le doute porte par exemple sur un projet professionnel jugé «pas sérieux», «non validé», "pas réfléchi en amont et préparé ». La demande différée comme stratégie routinière décrite plus haut vise alors à s'assurer du choix d'orientation annoncé par le jeune via un temps de réflexion, de démarche et de prise d'informations sur le métier («pour que le jeune puisse mûrir son projet »). Une forme de pointillisme bureaucratique, consistant à multiplier les demandes de pièces justificatives auprès d'un jeune, peut aussi servir à tester la réelle motivation des jeunes à obtenir cette aide et donc, dans l'esprit des professionnels, son caractère absolument nécessaire et une bonne probabilité de succès.

Dans le champ du handicap, la détection des demandes abusives se fait en équipe 
pluridisciplinaire ou peut, en cas de doute, être reportée en commission. Ainsi, les membres des commissions suspectent parfois de fausses déclarations (" ce sont de gros bobards ») ou relèvent une attitude particulièrement désinvolte et irréaliste : «Elle a coché toutes les cases, elle a fait ça au petit bonheur la chance!». Le tri se fait aussi en fonction du pronostic posé sur le bénéfice que l'individu pourra retirer de l'aide octroyée. La commission peut ainsi décider de limiter le nombre d'heures d'aide humaine accordées pour préserver l'autonomie de la personne et limiter les risques d'enfermement dans le handicap et de stigmatisation. Les débats autour des conséquences néfastes de l'aide sur le développement des personnes surgissent particulièrement au sujet des enfants intégrés en milieu scolaire. Les membres des commissions sont en outre conduits à faire la distinction entre les projets socialement et collectivement soutenables et ceux qui, bien que peu condamnables moralement, relèvent d'un choix personnel qui n'a pas à être suivi par la collectivité. Nous avons observé des cas où la délibération aboutit au refus de prendre en charge des coûts considérés comme la conséquence d'un choix délibéré de la personne handicapée, par exemple des frais de taxi pour une mère de famille malvoyante qui s'est installée dans une commune excentrée et mal desservie par les transports en commun, ou le coût d'une formation en langue des signes pour une jeune étudiante malentendante mais oralisée, dont l'intérêt pour la langue des sourds relève plutôt d'un projet professionnel et militant. On retrouve ici l'idée que l'aide doit être strictement nécessaire et ne doit pas répondre à des choix personnels, vus comme étant de l'ordre du confort.

Le même type de raisonnement prévaut dans les commissions FAJ lors de demandes d'aides financières au projet de formation. Les deux commissions observées s'accordent pour ne pas financer des projets de réorientation professionnelle de jeunes ayant déjà obtenu une qualification dans un secteur qui n'est pas sinistré. La formation envisagée n'est pas alors, selon eux, une mesure de correction nécessaire d'un handicap sur le marché du travail ; elle est l'expression d'un désir de changement ou d'une lassitude qui n'engage que l'individu et dont la société ne peut pas être solidaire.

\section{$\underline{\text { 2.3. La construction des frontières de l'aide par la jurisprudence }}$}

Afin de permettre en théorie une adaptation aux situations singulières de chaque individu, les règles d'attribution des aides financières demeurent volontairement floues. Cette indétermination amène régulièrement les membres des commissions à s'interroger sur le périmètre d'intervention qui est le leur. Chaque décision sur un cas engage quelque peu la philosophie d'ensemble du dispositif à travers la construction progressive, chemin faisant, 
d'une norme de solidarité publique.

Concernant les limites à donner à la compensation du handicap, certains points font l'objet d'une jurisprudence assez détaillée. Par exemple, un guide de jurisprudence, élaboré dans l'une des MDPH observées, précise que l'aide humaine accordée dans le cadre de la prestation de compensation ne couvre pas les actes liés à la parentalité. En cohérence avec cette doctrine, un homme atteint d'une maladie neurologique et séparé de sa femme s'est vu refuser le financement d'une aide à temps plein qu'il estimait pourtant nécessaire pour « le mettre en capacité de s'occuper de ses enfants »; dans un autre département, un jeune homme, handicapé à la suite d'un traumatisme crânien, n'a pas obtenu le titre d'aidant familial qu'il demandait pour sa compagne, contrainte de lui porter assistance pour ses troubles de mémoire et pour l'aider à porter son enfant malgré des pertes d'équilibre. Si la prestation de compensation du handicap est financée par le conseil général sur une enveloppe non fermée, les équipes tendent systématiquement à se replier prudemment vers des décisions d'aide « a minima », intégrant le risque d'explosion des dépenses.

A la lisère entre la question des frontières du champ et la question de l'abus, on rencontre l'argument le plus fréquemment utilisé pour écarter une demande : «c'est un problème social, ça ne relève pas de la $M D P H »$. Pour autant, ce type de jugement ne fait pas consensus, tant la frontière entre domaine du handicap et champ du social reste imprécise et sujette à discussion. Les divergences reflètent et prolongent une tension inhérente à la loi - et aux débats qui l'ont précédé - entre les deux définitions concurrentes du handicap : d'une part, une conception médicale, substantialiste ; d'autre part, une conception globale, attachée à la prise en compte de l'environnement et des « situations de handicap » (Bureau et Rist, 2011).

Dans l'insertion des jeunes, on note une grande disparité dans la prédéfinition des domaines de l'aide. Les départements balisent plus ou moins le champ d'intervention dans leur règlement intérieur ; les deux départements sur lesquels nous avons enquêté offrent deux formes d'investissement radicalement opposées, avec d'un côté un simple recto-verso qui n'ajoute guère de précisions par rapport à la loi et de l'autre un document d'une dizaine de pages qui liste des conditions spécifiques par domaines d'intervention. Il n'en reste pas moins que les limites du champ d'intervention s'éprouvent dans le traitement des dossiers singuliers, notamment parce que diverses dérogations «à titre exceptionnel et en fonction de la situation ", sont les codicilles courant de certains critères des règlements. Telle jeune fille demande une aide pour le passage du permis B alors qu'elle est en CDI mais sa conseillère craint que le temps de transport en commun qu'elle assure quotidiennement pour se rendre sur 
son lieu de travail ne finisse par compromettre son maintien en emploi. La définition de la précarité que l'on retient (uniquement fondée sur le type de contrat ou élargie à d'autres indicateurs) détermine l'évolution des normes de la solidarité publique. De même le maintien en emploi relève-t-il des missions de l'insertion? L'enjeu n'est pas seulement politique ou philosophique, il est aussi financier car les décisions d'attribution se prennent ici dans le cadre d'une enveloppe financière fermée qui suppose une maîtrise des coûts à tous les instants. Concernant les FAJ projet on peut prouver statistiquement que les décisions sont sensibles à l'enveloppe budgétaire disponible : sur le FAJ A, lorsque les professionnels ont la certitude que le reliquat de budget suffira largement à finir l'année (deux derniers mois de l'année d'exercice 2010), le taux de refus des aides chute de 10,5 points (de $24,3 \%$ à $13,8 \%$ ).

Ce type de dispositif au droit peu codifié est très sensible à la jurisprudence. La question est de savoir si le cas peut être jugé exceptionnel ou bien est susceptible de devenir la norme, au risque d'une dérive financière impossible à contenir :

«On ne va pas financer le permis de tous les jeunes [...] On a dit non pour une même situation, pour un emploi d'assistante commerciale, pourquoi on dirait oui sur cette situation là ?» (présidente de commission, FAJ A, l'aide est refusée).

«Attention, parce que là ça veut dire qu'on est prêt à financer tous les jeunes sans expérience qui sortent de CFA [...] Est-ce bien une situation marquée par une difficulté particulière ? » (présidente de commission, FAJ B, l'aide est refusée).

\section{Quelle place pour les usagers ? Stratégies et interactions.}

Malgré les injonctions à la prise compte des situations singulières, les deux dispositifs étudiés s'inscrivent dans une logique à dominante bureaucratique, adaptée à un traitement individualisé de masse : nos observations mettent en évidence la faible participation effective des usagers dans ces dispositifs qui traitent essentiellement les situations sur dossiers, (montés qui plus est par d'autres que le bénéficiaire, dans le cas du FAJ). Toutefois, des marges de manœuvre existent, qui laissent une place à l'interaction avec les demandeurs.

\subsection{Une expression de résistance dans le FAJ}

Dans le cas du FAJ, l'asymétrie d'information joue fortement : les règlements intérieurs ne sont pas diffusés, les jeunes ignorant souvent l'existence du dispositif ; les jeunes ignorent notamment que le professionnel à l'obligation d'instruire une demande relevant de l'aide sociale.

Dans ce contexte, les jeunes tentent d'exprimer leur volonté dans des stratégies plutôt 
défensives ou de résistance face aux injonctions des acteurs professionnels et institutionnels. Nous avons vu aussi à travers les catégories des conseillers que les jeunes peuvent faire pression sur les instructeurs pour qu'ils instruisent la demande ${ }^{1}$. Dans d'autres cas les jeunes peuvent décider de ne pas donner suite. La demande peut avorter des le départ lorsque le demandeur juge trop élevé le coût symbolique imposé par les contraintes du dispositif (Mazet, 2010), en raison des contraintes, des justifications «privées » à fournir, des délais, du sentiment d'avoir peu de chances d'obtenir quelque chose. Les instructeurs soulignent combien nombre de jeunes ne donnent pas suite et ne reviennent pas avec les justificatifs après avoir eu des informations sur la procédure FAJ et un avis sur leur faibles chances d'obtention d'une aide.

Enfin, les jeunes peuvent poser un recours s'ils contestent le refus d'aide de la commission. Si les recours sont rares, ils présentent néanmoins l'intérêt d'offrir, sur l'un des deux départements, un espace au bénéficiaire pour défendre son dossier sans filtrage préalable. La procédure offre ainsi au jeune la possibilité d'être présent en commission pour présenter son recours. Cela reste cependant une modalité de participation limitée et encadrée, car les commissions considèrent que le recours du jeune doit apporter des éléments nouveaux ayant un rapport avec le motif de refus initial posé. Ainsi le jeune qui fait recours participe, en théorie, uniquement comme «donnée» servant à la décision, comme pourvoyeur de paramètres informationnels nouveaux et corrects; on n'attend pas de lui un récit général du malheur. Pourtant, le dialogue engagé permet parfois d'influencer favorablement l'examen du recours ${ }^{\text {viii. }}$ C'est le cas pour une demande de financement du permis de conduire présentée par une jeune fille, qui en a besoin pour poursuivre son BTS de commerce. Dans un premier temps, la commission a refusé le financement, au motif que ses ressources étaient supérieures au barème prévu. Mais l'audition révèle une situation familiale très difficile (parents malades) et des effets de malchance avec le vol du véhicule du conjoint. La jeune fille, sur le point de pleurer en racontant ses difficultés, produit une forte émotion durant l'échange. Elle précise que si elle n'obtient pas l'aide du FAJ, elle prendra un crédit pour financer son permis. La confrontation avec la détresse de la jeune fille durant l'audition fait en définitive basculer la décision, au nom d'une série d'arguments qui ont en fait peu de rapport avec le critère officiel d'examen des recours : «Le projet professionnel tient la route » dit un éducateur. "C'est rare, des jeunes qui viennent défendre leurs dossiers » souligne un autre. "Elle a parlé de

\footnotetext{
${ }^{1}$ Ce type de données d'enquête indirectes rend impossible l'identification des caractéristiques sociales des jeunes qui « insistent» comparativement à celles de ceux qui se «découragent» rapidement face aux contraintes du dispositif.
} 
prendre un crédit [s 'il n'y a pas de financement FAJ et pour payer une voiture], ça va être la catastrophe » estime une assistante de service social. Enfin une conseillère en mission locale insiste sur le fait que sans l'obtention du permis, elle ne pourra pas poursuivre son BTS.

\subsection{L'organisation de l'expression des usagers dans les MDPH}

On peut observer, dans les MDPH, des constats semblables de renversement de situations. Si elles ne concernent qu'une faible part des dossiers traités, les auditions n'en constituent pas moins un moment clé du processus d'attribution, durant lequel les personnes ont la possibilité de contester les jugements émis sur leurs situations et de modifier la décision.

Les deux MDPH que nous avons observées partagent le souci d'aménager des conditions d'écoute qui favorisent l'échange. L'une d'entre elles reçoit les personnes en commission restreinte, afin de préserver un cadre moins intimidant, propice au dialogue. Le guide de jurisprudence de la seconde précise, de son côté, que l'audition peut se faire en deux temps si la personne est accompagnée, « et ce afin de veiller à conserver un instant de dialogue seul». Il s'agit de s'assurer que l'expression du besoin n'est pas déformée ou empêchée par des intérêts des proches, parfois divergents de ceux du demandeur. L'enjeu des familles, peu présent dans le cas des FAJ, s'avère en effet central dans le champ du handicap, où l'entourage est souvent fortement mobilisé.

Lors des auditions en CDA, les usagers mobilisent quatre principaux registres d'expression pour expliquer leur situation et «défendre leur cas »: la mise en scène du handicap, l'argumentation à partir de données factuelles, l'appel au sens moral ou la revendication d'une reconnaissance du mérite (Bureau et Rist, 2012). Ces registres recouvrent en partie ceux qu'évoque Didier Fassin (2000) dans son étude des stratégies rhétoriques utilisées dans les courriers de demandes d'aide d'urgence : la compassion, la nécessité, la justice, le mérite. Ces différents registres ne sont pas tous aussi fructueux. A cet égard, nous rejoignions les constats de D. Fassin, qui montre que l'indignation ou l'appel au mérite sont rarement récompensés. La mise en scène dramaturgique ou l'apport d'explications nouvelles sur sa situation peuvent en revanche faire basculer les décisions dans un sens favorable à l'usager. A ces différentes figures de participation individuelle des usagers en commission s'ajoutent les modes d'expression indirects et collectifs des usagers, par le biais des associations qui les représentent. Il est clair que la possibilité d'être représenté ou non constitue un facteur de différenciation majeur entre les droits d'expression des jeunes dans le FAJ et les personnes handicapées en commission. Les associations d'usagers interviennent selon cinq modes principaux. Ils peuvent contester l'expertise professionnelle de l'équipe pluridisciplinaire, au 
nom d'un savoir du vécu, d'une expertise du proche; plaider la cause des personnes handicapées, sur un mode empathique, en se faisant l'avocat de la personne handicapée; jouer un rôle de vigilance éthique, en tant que simples citoyens, garants du respect des règles éthiques et détenteurs d'une mémoire jurisprudentielle ; donner un avis de sens commun, au nom d'un savoir commun sur les situations de la vie courante et d'un sens ordinaire de la justice; ou encore, plus rarement, défendre une philosophie globale de l'intégration des personnes handicapées, sans prendre appui sur une expertise particulière ni plaider la cause de telle ou telle.

Plusieurs raisons militent pour une connaissance fine des besoins des personnes handicapées et justifient l'attention mobilisée par les équipes en commission, pour comprendre l'analyse que les demandeurs ou leurs représentants font de leur vulnérabilité. Outre l'importance des montants en jeu, et le poids des associations représentatives des usagers dans les débats, chacun mesure les risques potentiellement tragiques d'une mauvaise orientation ou d'un retrait d'allocation pour une personne handicapée. Par ailleurs, la forte dimension compassionnelle liée au handicap et l'intensité dramaturgique de la confrontation directe aux personnes handicapées jouent évidemment un rôle essentiel. Pour ces raisons, la comparaison des deux dispositifs permet de faire l'hypothèse que l'expression directe des bénéficiaires sur leurs besoins est sensiblement plus valorisée dans les CDA que dans le cas du FAJ. Toutefois, il apparaît que les moments d'interaction observés, dans les deux dispositifs, constituent des espaces d'exception au sein du traitement bureaucratique des dossiers.

\section{Conclusion}

Notre approche comparée de la mise en œuvre des politiques sociales permet de nourrir une réflexion sur le non accès aux droits et la construction de la citoyenneté sociale moderne. Elle montre que l'individualisation des politiques publiques et notamment des politiques sociales ne favorise pas mécaniquement une participation accrue des usagers. Ainsi dans le traitement individualisé de masse en matière d'aide sociale, il semble abusif de parler de co-construction négociée et individualisée des besoins et des projets. En ce sens la dimension procédurale du droit, un droit supposé ouvert et indéfini a priori, ne crée pas pour autant de la coconstruction. Le cas du FAJ illustre une politique extrêmement personnalisée, située, structurée par l'injonction à l'autonomie et néanmoins marquée par l'éviction quasi-totale des usagers du circuit de décision puisqu'ils n'ont même pas la liberté de choisir le moment du dépôt de la demande d'aide financière. Par contraste le cas du handicap montre que 
l'expression des usagers doit être pensée dans l'agencement et dans les procédures des dispositifs pour être effective et contrebalancer l'asymétrie structurelle de la relation d'aide.

On souligne aussi en mettant en parallèle ces deux dispositifs combien la question de la participation individuelle des usagers est indissociable d'une réflexion sur la représentation collective de leurs intérêts. L'impensé de la représentation des intérêts qui caractérise les politiques sociales et économiques de jeunesse à tous les niveaux de l'action publique (Lima, $2010 \mathrm{~b}$ ) conforte les gens de terrain dans leur attitude paternaliste vis-à-vis des jeunes pris individuellement. L'absence de mouvements autonomes de la jeunesse précaire et plus encore l'impossibilité organisée par le dispositif d'un porte-parolat des jeunes demandeurs laissent libre-cours à l'expression de l'archétype du bénéficiaire valide et apte au travail traditionnellement plus contrôlé par les institutions du travail social. Au contraire dans le cas du handicap on peut penser que la présence d'associations d'usagers favorise le déploiement d'une variété plus grande d'expression des demandeurs. Le souci de la représentation collective dans les décisions d'octroi de droits est d'autant plus crucial que la « politique du guichet », qui fait de l'interaction entre le streetlevel bureaucrats et l'usager « le lieu sur lequel repose le mode opératoire d'une intervention publique visant à réguler ces populations » (Dubois, 2010b), est prolongée par une « politique de la commission » qui met en délibération les cas individuels dans une arène constituée d'acteurs tout à la fois représentants (de leur institution, de leur profession, de l'Etat) et experts. Cette mise à distance relative de la décision par rapport au moment de l'interaction au guichet oblige à reposer la question de la participation des usagers dans l'ensemble de la chaîne de traitement.

\section{Bibliographie}

ASTIER I. (1997), Revenu minimum et souci d'insertion, Paris, Ed. Desclée de Brouwer.

BUREAU M-C., RIST B. (2012) : «Expertise, délibération et dramaturgie dans l'attribution des droits sociaux liés au handicap ", in ABALLEA F. (dir.), Institutionnalisation, désinstitutionalisation de l'intervention sociale, Toulouse, Octarès, p. 249-262.

BUREAU M-C., RIST B. (2012): «Les figures de la participation des usagers dans les commissions d'attribution des droits aux personnes handicapées », Vie sociale $n^{\circ} 4 / 2012$, p. 81-98.

DUBOIS V. (2010a) : La vie au guichet. Relations administratives et traitement de la misère, Paris, Economica, 3ème édition.

DUBOIS V. (2010b) : «Politiques au guichet, politiques du guichet», in BORRAZ O. et 
GUIRAUDON V. (dir.), Politiques publiques 2. Changer la société, Paris, Presses de Sciences Po, p. 265-286.

FASSIN D. (2000) : «La supplique. Stratégies rhétoriques et constructions identitaires dans les demandes d'aide d'urgence ». In Annales. Histoire, Sciences Sociales. 55e année, N. 5, pp. 955-981.

GUIRAUDON V., Politiques publiques 2, Changer la société, tome 2, Presses de SciencesPo, p. $265-286$.

GENEST S., GORGEON C., LEON H., VERDIER R. (2003), « L'organisation et la mise en œuvre des fonds d'aide aux jeunes - Analyse comparée dans cinq départements », DREES, Études et Résultats, $\mathrm{n}^{\circ} 221$.

LIMA L. (2008), « Le temps de la prime insertion professionnelle : un nouvel âge de la vie », in Guillemard A-M. (dir), Où va la protection sociale?, Paris, PUF, p. 49-67.

LIMA L. (2010a), «L'expertise sur autrui comme nouveau mode de régulation de la protection sociale. Principes et dispositifs », Working Papers RT6, 2010-1, 13 p.

LIMA L. (2010b), «Les différences de situations entre générations dans le débat public », Regards croisés sur l'économie, $\mathrm{n}^{\circ}$ 7, mai, p. 71-79.

MARTUCELLI D. (2004), «Figures de la domination », Revue française de sociologie 45-3, p. $469-497$.

MAZET P. (2010), « La non demande de droit : prêtons l'oreille à l'inaudible », La vie des idées, http://www.laviedesidees.fr/La-non-demande-de-droits-pretons-1.html

NIVIERE D. (2006), « Les fonds d'aide aux jeunes : Bilan de l'année 2004 à la veille de la décentralisation du dispositif », DREES, Etudes et résultats, $n^{\circ} 493$.

ROSANVALLON P. (1995) : La nouvelle question sociale, Paris, Seuil.

SIBLOT Y. (2006), Faire valoir ses droits au quotidien. Les services publics dans les quartiers populaires, Paris, Les Presses de Sciences Po.

SPIRE A. (2008), Accueillir ou reconduire. Enquête sur les guichets de l'immigration, Paris, Raisons d'agir.

VIDAL-NAQUET P.A. (2009), “Quels changements dans les politiques sociales aujourd'hui? Le projet entre injonction et inconditionnalité", La nouvelle revue de l'adaptation et de la scolarisation, $\mathrm{n}^{\circ} 47$, p. 1-16. 


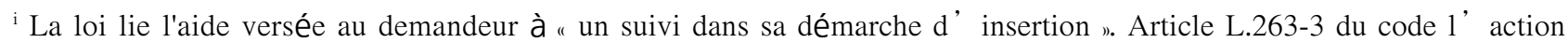
sociale et des familles. Les départements ont tendance à interpréter cette disposition comme une condition préalable au bénéfice de 1' aide.

ii En témoigne la liste suivante : accueil de la petite enfance, scolarité, enseignement, éducation, insertion professionnelle, aménagements du domicile ou du cadre de travail, développement ou aménagement de l'offre de service, développement de groupes d'entraide mutuelle ou places en établissements spécialisés, aides de toute nature à la personne ou aux institutions pour vivre en milieu ordinaire ou adapté, etc. (Article L114-1-1 créé par Loi n ${ }^{\circ} 2005-102$ du 11 février 2005 - art. 11 JORF 12 février 2005)

iii MDPH An III, La vigilance, Rapport d’ activité 2008 des Maisons Départementales des Personnes Handicapées, 2009, CNSA.

iv Par exemple, la mesure de diminution de 1' acuité visuelle des deux yeux détermine un taux médical d'incapacité.

${ }^{v}$ Le GEVA, mis en place par le décret du 6 février 2008, constitue la référence nationale pour l'évaluation des besoins de compensation des personnes handicapées. Il ne sert pas à mesurer le taux d'incapacité des personnes handicapées mais il est destiné à aider les équipes pluridisciplinaires à élaborer un plan personnalisé de compensation (PPC) adapté aux besoins spécifiques de la personne.

${ }^{v i}$ A titre indicatif, l'une des CDA observées auditionne en moyenne 400 à 450 usagers par an sur environ 30000 dossiers.

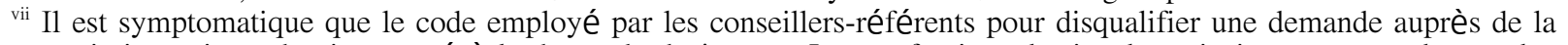
commission soit : " dossier monté à la demande du jeune ». Les professionnels signalent ainsi que, non seulement la demande a été spontanément formulée par le jeune mais aussi qu' il a passé outre 1' opposition du référent qui désapprouve cette démarche.

viii Dans 2 cas sur les 4 observés, la commission a changé d'avis, suite à l'impression positive faite par les jeunes reçus. 